\author{
EVS27 \\ Barcelona, Spain, November 17-20, 2013
}

\title{
Predicting lithium-ion battery degradation for efficient design and management
}

\author{
Sébastien Grolleau ${ }^{1,2}$, Arnaud Delaille ${ }^{1}$ Hamid Gualous ${ }^{2}$ \\ ${ }^{1}$ Laboratory for Electrical Storage, CEA//LITEN//DTS/LSE, 73377 Le-Bourget-du-Lac, France, \\ sebastien.grolleau@,cea.fr \\ ${ }^{2}$ Université de Caen Basse Normandie, LUSAC Normandie rue Louis Aragon, 50130 Cherbourg-Octeville, France
}

\begin{abstract}
Being able to predict the Li-ion battery degradation is necessary for applications such as electric vehicles (EVs) and hybrid ones (HEVs). Most of the time, battery life prediction is based on accelerated cycling datasets obtained under different conditions. However, cell aging occurs not only during cycling but also at rest (calendar mode), the latter representing about $90 \%$ of its lifetime. In this work, an empirical model of a $12 \mathrm{Ah}$ commercial graphite/nickel-manganese-cobalt (C/NMC) cell accounting for calendar aging is presented. An innovative accelerated aging protocol representative of a battery usage likely to be encountered in real-world is also proposed. Experimental results tend to prove that a state-of-charge (SoC) range management can extend the battery lifetime significantly, mainly due to the calendar aging effect. Furthermore, results show that even a low battery usage, limited to $10 \%$ of the total time, has a detrimental effect on the cell lifetime that a pure calendar aging model is unable to predict.
\end{abstract}

Keywords: lithium battery, battery calendar life, battery management, modelling

\section{Introduction}

Lithium-ion battery (LIB) technology is a promising candidate for automotive applications due to its impressive energetic and power densities. However, long cell lifetime is still uncertain for LIBs. Cell aging occurs during both cycling and storage modes (the latter being called calendar mode). Considering that a personal car spends about $90 \%$ of its life in parking mode, studying LIB aging during storage seems particularly relevant. In addition, predicting efficiently lithium-ion cells degradation is necessary for lifetime evaluation, especially in applications such as EVs and HEVs for which lifespans of 10 to 15 years are desired [1].
The paper is organized in two parts. Section 2 describes the capacity fade prediction model and regression of model parameters to experimental data obtained on a commercial 12 Ah LIB. Section 3 provides aging results of identical cells under an innovative aging protocol, representative of realoperating conditions in terms of charge, discharge, and rest time periods, except the fact that the temperature is set to $45^{\circ} \mathrm{C}$ in order to accelerate aging phenomena.

\section{Capacity fade model}

\section{$2.1 \quad$ Tested cells}

Aging experiments were conducted on similar commercial prismatic 12 Ah graphite and nickel- 
based oxide (C/NMC) cells. The cell ratings given by the cell manufacturer are summarized in Table1.

Table1: cell characteristics

\begin{tabular}{|l|c|}
\hline Parameters & Values \\
\hline Nominal capacity at $1 \mathrm{C}_{\text {nom }}, 25^{\circ} \mathrm{C}(\mathrm{Ah})$ & 12 \\
\hline End-of-discharge voltage $(\mathrm{V})$ & 2.70 \\
\hline End-of-charge voltage (Floating) $(\mathrm{V})$ & 4.20 \\
\hline $\begin{array}{l}\text { End-of-charge current under floating } \\
\text { voltage (A) }\end{array}$ & $\begin{array}{c}0.6 \\
(\mathrm{C} / 20)\end{array}$ \\
\hline
\end{tabular}

Cell were charged with a classical Constant Current / Constant Voltage $(\mathrm{CC} / \mathrm{CV})$ charge up to $4.20 \mathrm{~V}$ and hold until a cut-off current of $I_{\text {cut }}$. off $=\mathrm{C}_{\mathrm{nom}} / 20$ is reached.

\subsection{Calendar aging data}

Aging datasets upon storage under static conditions (at rest) were obtained in the framework of the SIMCAL project [2]. The SIMCAL research program aims to study and to understand the calendar aging mechanisms of a wide variety of new battery technologies since these mechanisms are still unclear (especially for new generation of LIBs).

Two stress factors have been investigated: the State of Charge (SoC) and the temperature of storage. Three $\mathrm{SoC}$ and three temperatures are considered, respectively $30 \%, 65 \%, 100 \%$ and $30^{\circ} \mathrm{C}, 45^{\circ} \mathrm{C}, 60^{\circ} \mathrm{C}$. During storage, a substantial part of the capacity loss can be recovered after a full charge using the $\mathrm{CC} / \mathrm{CV}$ protocol defined in Error! Reference source not found.. Therefore, three discharge/charge cycles at $1 \mathrm{C}_{\text {nom }}$ at $25^{\circ} \mathrm{C}$ were performed. At the end of each performance characterisation test $(\mathrm{CT})$, a $1 \mathrm{C}_{\text {nom }}$ discharge was applied to the fully charged cells in order to reset the $\mathrm{SoC}$ before storage.

\subsection{Model description}

The capacity prediction model is fit using robust nonlinear regressions conducted on calendar aging data obtained in the nine static conditions.

Loss of cyclable lithium has been identified as the dominant aging mechanism as a result of parasitic reactions at the electrolyte/electrode interface, especially for graphite based anodes, during both cycling and calendar aging $[3,4]$. In this work, an empirical expression accounting for both diffusive and kinetic limitations is proposed:

$$
\frac{d Q_{\text {loss }}}{d t}=\frac{k(T, S o C)}{1+A \cdot\left(Q_{\text {loss }}+Q_{0}\right)}
$$

The term $k(T, S o C)$ encapsulates both $T(K)$ and $S o C$ kinetic dependencies as follows:

$$
\begin{aligned}
& k(T, S o C)=\exp \left(a_{S o C} \cdot S o C+b_{S O C}\right) . \\
& \exp \left(-\frac{a_{T} \cdot S O C+b_{T}}{T}\right)
\end{aligned}
$$

Parameters $A, Q_{0}, a_{S o c}, b_{S o c}, a_{T}$ and $b_{T}$ are fixed and derived from calendar aging experimental data as described below. Fig. 1 compares capacity loss evolution with storage time with model regressed for each aging condition.

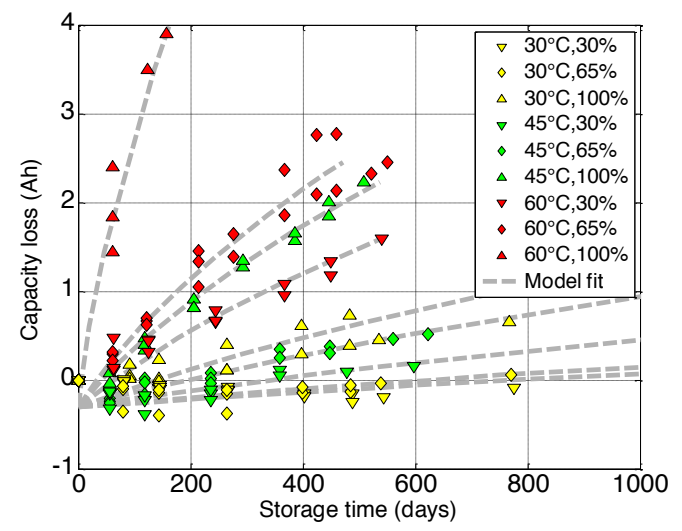

Figure1: Capacity loss under storage at various temperatures and SoCs fitted with the aging model.

\section{Calendar aging under dynamic conditions}

\subsubsection{Battery cycle profiles}

Beside static storage conditions, six cells were submitted to three different cycling profiles (Fig. 2 ). The cycling profiles are based on a simplified driving usage of a pure electrical urban vehicle which is used twice a day, simulating a daily commuting between home and workplace. Furthermore, we have considered an EV with 150 $\mathrm{km}$ (around 95 miles) of full autonomy, and some travel distances of $30 \mathrm{~km}$ (around 19 miles) corresponding roughly to $20 \%$ of $\mathrm{SoC}$ in our case. Each cycling profile corresponds to a specific charging scenario: in scenario 1, the battery is fully recharged every night, following immediately the second trip of the day. As a consequence, the battery is operated in the SoC range $100 \%-60 \%$, with one cycle per day. In scenario 2 , the battery is fully recharged only when the battery SoC reaches $20 \%$, with one cycle in two days. The scenario 3 is the same as the scenario 1 except that the battery is operated between the intermediate $\mathrm{SoC}$ range $60 \%-20 \%$. 
Both discharging and charging rates are constant and have been fixed respectively to $\mathrm{C}_{\text {nom }} / 2$ and $\mathrm{C}_{\text {nom }} / 5$ in all cycling profiles considered in this work. The discharge time has been set to 30 minutes. For scenarios 1 and 2 , the charging protocol is the standard $\mathrm{CC} / \mathrm{CV}$ protocol. The end-of-charge cut-off current is the same value as in Error! Reference source not found.. For scenario 3 , the charging time at the end of the day is set to 60 minutes. We can underline here that at the end of the second day, the amount of charge removed from the battery is the same for each charging scenario.

Ambient temperature was fixed to $45^{\circ} \mathrm{C}$ in order to accelerate aging tests. Each cycling profile is repeated continuously during four weeks. Every month, cycling is interrupted and capacity fade is monitored by constant current discharges performed at $25^{\circ} \mathrm{C}$. Although simplistic, our approach respects the repartition between calendar and cycling aging conditions expected in a real-world environment. Indeed, $87 \%$ of the aging time is spent at rest for all scenarios, which is a typical value for an EV battery.

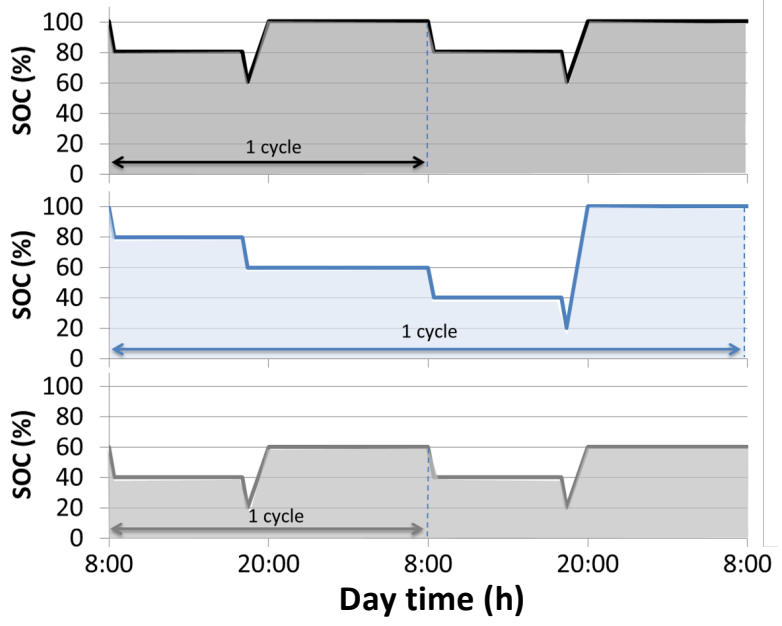

Figure2: SoC patterns of the three considered scenarios. Discharging and charging rates has been fixed respectively to $\mathrm{C}_{\text {nom }} / 2$ and $\mathrm{C}_{\text {nom }} / 5$.

\subsubsection{Experimental results}

Fig. 3 shows measured State of Health $(\mathrm{SoH})$ or relative capacity during aging under each battery cycling profiles described in 3.1.1. Relative capacity evolution of the static aging condition $45^{\circ} \mathrm{C}, 100 \%$ SoC (corresponding to a pure calendar aging) are also displayed, from results obtained in the SIMCAL project as described in 2.2 .

Charging scenarios 1 and 2 exhibit a very substantial effect on battery degradation evolution. Cells submitted to the scenario 3 have lost $6 \%$ of their nominal capacity after 10 months of aging. In the meanwhile, cells submitted to scenario 1 have lost $25 \%$ of their nominal capacity. Furthermore, scenario 1 leads to a rapid capacity decline after seven months. The cells recharged every day spend a lot of time at high SoC, which is known to be very harmful for LIBs $[5,6]$.

By linearly extrapolating these preliminary results, battery end-of-life (defined by an $80 \% \mathrm{SoH}$ ) was reached after 9 months for scenario 1 , and expected to be reached after 14 months for scenario 2 and 3 years for scenario 3 .

Besides, it is interesting to notice that aging rates of charging scenarios 1 and 2 are both substantially higher than the one in static condition $\left(45^{\circ} \mathrm{C}\right.$, $100 \% \mathrm{SoC}$ ). On a calendar perspective, aging of both charging scenarios should rather be lower than the static condition, as cells spent less time at elevated SoC. The observed difference is hence a consequence of the cycling aging. Moreover, capacity fade of charging scenario 1 tends to accelerate substantially after six months of aging, and the discrepancy between the two tested cells is also much higher than the two other scenarios.

These results tend to prove that even a minimal usage can accelerate battery degradation. The following discussion tends to confirm this observation.

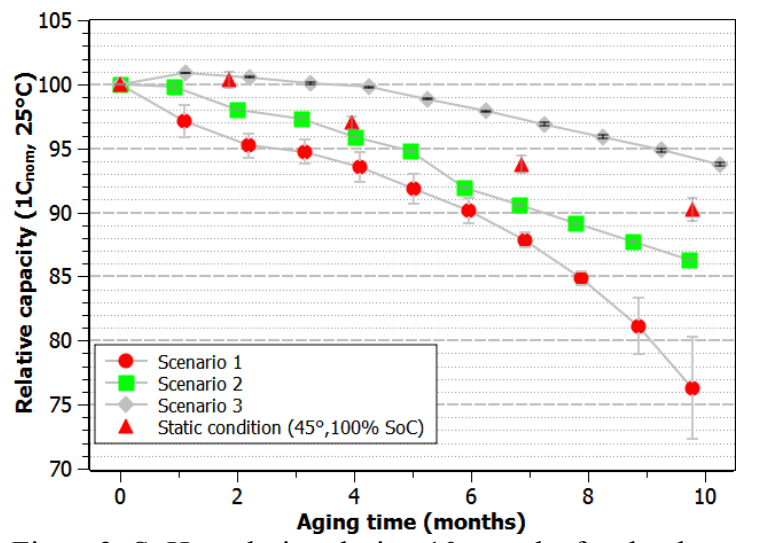

Figure3: SoH evolution during 10 months for the three scenarios ${ }^{1}$. Aging static conditions $\left(45^{\circ} \mathrm{C}, 100 \% \mathrm{SoC}\right)$ is also displayed. Error bars represent discrepancy between cells.

A first explanation could be a higher temperature of the cell during cycling. Indeed, periodic partial discharges and charges performed during each scenario could increase the cell temperature due to

\footnotetext{
${ }^{1}$ Aging tests under non static conditions were started in May 2012 and are still running. Therefore, at least one year aging results are expected for the date of the EVS27 conference.
} 
internal resistance. High temperatures, especially above $45^{\circ} \mathrm{C}$, have a strong detrimental effect on cell lifetime. Furthermore, the cell resistance tends to increase due to aging, which would contribute to accelerate the degradation and would explain results obtained for scenario 1. Surface temperatures of each individual cell were monitored using a K-type thermocouple. Temperature evolution over one day of a cell submitted to cycling scenario 1 is plotted against time along with cell potential on Figure4. If discharge and charge periods are clearly visible on the voltage profile, cell temperature remains however unaffected by battery usage. Actually, this can be explained by the relative low current rate used for all the cycling profiles $\left(\mathrm{C}_{\text {nom }} / 2\right.$ and $\mathrm{C}_{\text {nom }} / 5$ during discharges and charges respectively). Moreover, the prismatic geometry facilitates heat transfer from the cell to the surrounding air. The suspected temperature rise is thus not a valid hypothesis explaining the faster degradation of cells submitted to cycling profiles.

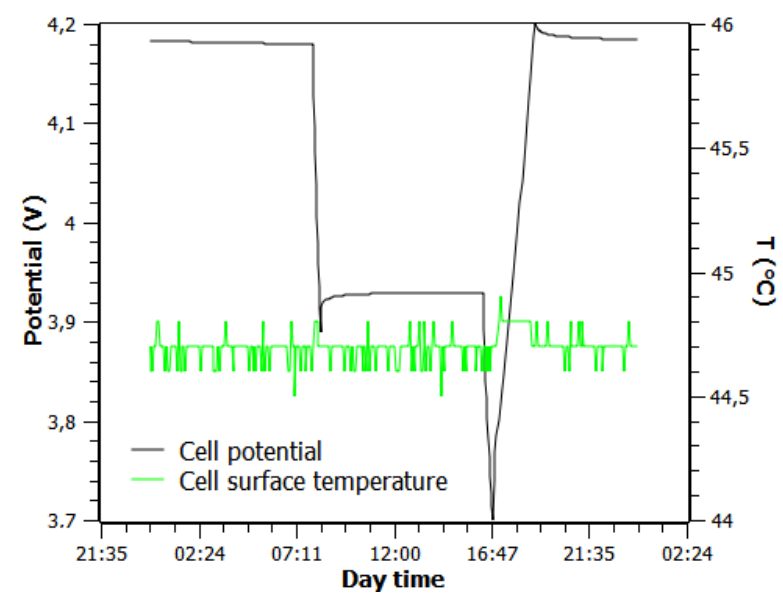

Figure4: Daily potential and temperature changes (cell submitted to cycling profile 1 after 10 months)

Another explanation could be the number of CTs performed on the cells submitted to the 3 cycling profiles.

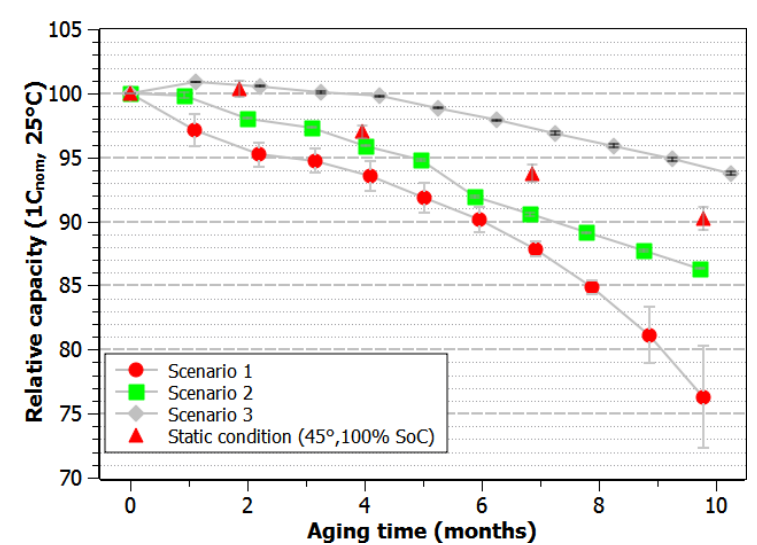

Figure 3 shows that this number is twice the one in static condition. Even though are performed at lower temperature $\left(25^{\circ} \mathrm{C}\right)$ than the rest of aging $\left(45^{\circ} \mathrm{C}\right)$, more frequent $\mathrm{CTs}$ may have a negative effect as they mean more cycles. Influence of the time between two consecutive CTs can also be investigated. Knowing that in the SIMCAL project, the time interval between two consecutive CTs was set to two months, we decided to study the capacity decay of cells submitted to the same static aging condition $\left(60^{\circ} \mathrm{C}, 100 \%\right.$ SoC) with one and two months between two consecutive CTs. It can be seen from Figure 5 that the interval between two consecutive CT has virtually no influence on capacity fade. This result clearly demonstrates that neither the number of CTs nor their frequency have an impact on the aging rate.

$60^{\circ} \mathrm{C}, 100 \%$ SoC

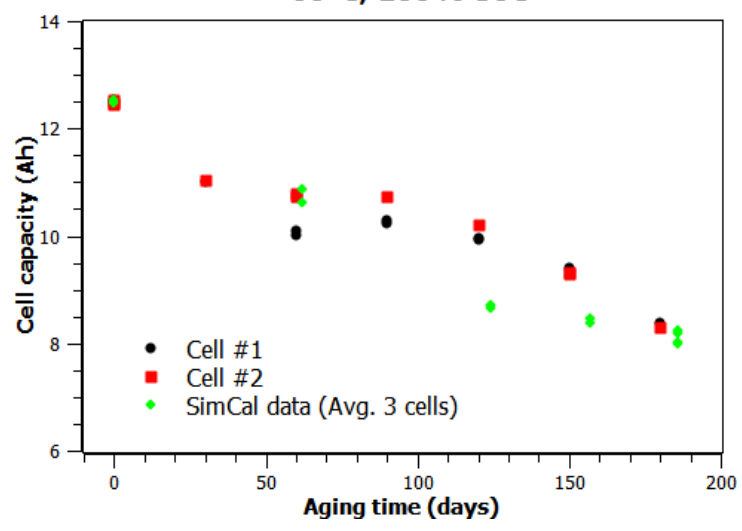

Figure 5: influence of time interval between two consecutive characterisation tests.

As both influence of temperature and number of CT were successively ruled out, our experimental results corroborate a negative impact of the battery usage on the cell lifetime. Aging results of realistic battery usage combining both calendar and cycling aging modes are rarely reported in the. In this work, although $87 \%$ of time is spent at rest, capacity fade of a realistic usage is considerably accelerated in comparison with the one induced by pure aging static condition. 
Moreover, the present work demonstrates that capacity fade is accelerated not only for a moderate battery usage but also for moderate charge and discharge current rates (charges at $\mathrm{C}_{\text {nom }} / 5$ and discharges at $\mathrm{C}_{\text {nom }} / 2$ ).

\section{Conclusion}

Simulating performance loss of LIBs is a fundamental step for design and commercialisation of EVs and HEVs. As most of the battery life is spent under storage, studying battery degradation during storage seems particularly relevant for this application. Launched in 2009, the SIMCAL research program has provided highly valuable calendar aging datasets, as well as in-depth analysis of aging phenomena of commercial high power lithium-ion cells.

In this work, a degradation model capturing temperature and state of charge was fit to calendar aging data of a $12 \mathrm{Ah}$ high power cell. Besides static calendar aging tests, complementary tests aging tests mixing both storage and usage conditions were performed. Aging protocol of these tests was specially selected to match a realistic repartition of an EV application where up to $90 \%$ of time is spent at rest. Temperature is fixed to $45^{\circ} \mathrm{C}$ to enhance aging phenomena.

As proven by our aging tests, battery degradation can be reduced not only by limiting battery temperature during cycling and storage, but also by avoiding frequent recharging. In addition, comparison between static and dynamic aging tests results tends to show that cycling has a strong negative impact on lifetime. Influences of temperature rise induced by cycling and test interruptions due to characterisation tests were consecutively discarded to explain the different aging rates between dynamic and static tests. As a consequence, even a moderate battery usage can a have a strong effect on capacity fade and cannot be neglected to make lifetime prediction for realistic EV usage. Physical analyses of the tested cells are already planned since they may help to fully understand the underlying phenomena.

\section{Acknowledgments}

The authors acknowledge funding of this work from University Caen Basse-Normandie and the Laboratory for Electrical Storage (CEA/LSE), operated by the French Alternative Energies and
Atomic Energy Commission. The authors are also grateful for the financial support of the SIMCAL project from the French National Research Agency and for helpful discussions with SIMCAL network partners.

\section{References}

[1] PHEV Batteries-Phase 2-RFPI-FINAL, http://www.uscar.org/commands/files downloa d.php?files id=217, accessed on 2013-02-12.

[2] M. Kassem, J. Bernard, R. Revel, S. Pélissier, F. Duclaud, C. Delacourt, Journal of Power Sources 208 (2012) 296.

[3] M. Safari, C. Delacourt, Journal of The Electrochemical Society 158 (2011) A1123.

[4] M. Broussely, S. Herreyre, P. Biensan, P. Kasztejna, K. Nechev, R.. Staniewicz, Journal of Power Sources 97-98 (2001) 13.

[5] M. Broussely et Al., Aging mechanism in Liion cells and calendar life predictions, Journal of Power Sources, 97-98(2001), 13-21

[6] J. Vetter, P. Novák, M.R. Wagner, C. Veit, K.C. Möller, J.O. Besenhard, M. Winter, M. Wohlfahrt-Mehrens, C. Vogler, A. Hammouche, Journal of Power Sources 147 (2005) 269.

\section{Authors}

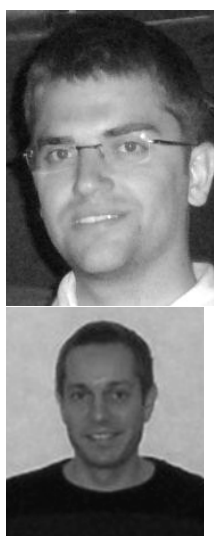

Sébastien Grolleau graduated from ISAE, Toulouse in 2008 as an engineer and holds a Research Master's degree in Fluid dynamics and Energetics. From 2008 to 2010, its activities concerned computer modeling of off-grid and gridconnected power systems including energy storage devices (fuel cell and batteries) and renewable sources. Since 2010, he pursues a Ph.D. at the French Alternative Energies and Atomic Energy Commission (CEA) in the Laboratory for Electrical Storage focusing on the aging upon storage of lithium-ion cells.

Dr. Arnaud Delaille, graduated of a $\mathrm{Ph} . \mathrm{D}$. of the University of Paris VI in 2006, electrochemistry specialty, about state-of-charge \& state-of-health indicators for lead-acid batteries used 
in stand-alone photovoltaic systems. Since 2006, he works as project manager at the French Alternative Energies and Atomic Energy Commission (CEA) in the Laboratory for Electrical Storage, involved in Battery Management System activities. He coordinates several projects, including the Project SIMCAL (2009-2012): French research network working on the modeling of calendar aging of batteries used in EVs and HEVs.

Hamid Gualous received his Ph.D. degree in electronic from the University Paris XI Orsay, France, in 1994. From 1996 to 2009 he was an Associate Professor at the University of Franche-Comté, FEMTO-ST Laboratory, France. Since 2009, he is Full Professor at the University of Caen Basse-Normandie LUSAC laboratory, France. His main research activities are concerning energy storage devices (supercapacitors and batteries), hybrid power sources (fuel cell-supercapacitor battery) and energy management for vehicle applications. 